\title{
Editorial
}

\section{Statistical and Probabilistic Approach in Monitoring-Based Structure Rating and Risk Assessment}

\author{
Ting-Hua Yi, ${ }^{1}$ Ying Lei, ${ }^{2}$ Hua-Peng Chen, ${ }^{3}$ Siamak Talatahari, ${ }^{4}$ and Fei Kang ${ }^{5}$ \\ ${ }^{1}$ School of Civil Engineering, Dalian University of Technology, Dalian 116023, China \\ ${ }^{2}$ School of Architecture and Civil Engineering, Xiamen University, Xiamen 361005, China \\ ${ }^{3}$ Medway School of Engineering, The University of Greenwich, Kent ME4 4TB, UK \\ ${ }^{4}$ Marand Faculty of Engineering, University of Tabriz, Tabriz 51368, Iran \\ ${ }^{5}$ School of Civil Engineering, Purdue University, West Lafayette, IN 47907, USA \\ Correspondence should be addressed to Ting-Hua Yi; yth@dlut.edu.cn
}

Received 26 June 2014; Accepted 26 June 2014; Published 14 July 2014

Copyright (C) 2014 Ting-Hua Yi et al. This is an open access article distributed under the Creative Commons Attribution License, which permits unrestricted use, distribution, and reproduction in any medium, provided the original work is properly cited.

Aging civil infrastructural facilities like long-span bridges, super-tall buildings, and large-scale space structures that form the life of a country's economy are facing a severe crisis in some countries. Long service lives, inadequate designs, and increasing extreme loads are responsible for the current state of affairs. Thus, knowledge about the in-service condition of the structures is one of the most essential parts required for the engineering community. This opens a wide field for structural health monitoring (SHM) systems which are set up to assure the safe operation of structures requiring linking sensors with computational tools able to interpret sensor data in terms of structural performance [1]. Although intensive development continues on innovative sensor systems, there is still considerable uncertainty in deciding structural behavior since there are many factors in abundant measured data from the SHM system that may influence the health assessment of a structure. The most appropriate and efficient way to alleviate this multiple input problem is by the statistical and probabilistic approach including data normalization, feature extraction, statistical modeling, and risk management [2-4].

Therefore, in the light of these considerations, this special issue was launched. Numerous investigators worldwide were invited to contribute their original papers and review articles on the theme of this special issue. A total of 27 technical papers are included in this special issue. These papers present the most recent advances, progress, and ideas in the field of the statistical and probabilistic approach and its application in structure rating and risk assessment includes data compression and cleaning, data mining and fusing technology, pattern recognition and feature extraction, damage detection and condition assessment, and performance prediction and risk management. All of the accepted papers were carefully reviewed and found appropriate for the journal.

Without a doubt, the papers reflect the state-of-the-art researches and developments of this subject.

\section{Acknowledgments}

As the guest editors of the special issue, we would like to express our sincere appreciation to all the authors who contributed their work to this exciting special issue. The guest editors also thank the reviewers for their valuable and insightful comments that greatly benefited the improvement of paper quality. This meaningful work was jointly supported by the National Natural Science Foundation of China (Grant nos. 51222806, 51121005, and 51327003) and the Specialized Research Fund for the Doctoral Program of Higher Education (Grant no. 20130041110031).

Ting-Hua Yi Ying Lei

Hua-Peng Chen Siamak Talatahari

Fei Kang 


\section{References}

[1] T.-H. Yi, H.-N. Li, and M. Gu, "Recent research and applications of GPS-based monitoring technology for high-rise structures," Structural Control and Health Monitoring, vol. 20, no. 5, pp. 649670, 2013

[2] T. Yi, H. Li, and M. Gu, "Characterization and extraction of global positioning system multipath signals using an improved particle-filtering algorithm," Measurement Science and Technology, vol. 22, no. 7, Article ID 075101, pp. 1-11, 2011.

[3] T. H. Yi, H. N. Li, and M. Gu, "Experimental assessment of high-rate GPS receivers for deformation monitoring of bridge," Measurement, vol. 46, no. 1, pp. 420-432, 2013.

[4] T.-H. Yi, H.-N. Li, and H. M. Sun, "Multi-stage structural damage diagnosis method based on "energy-damage" theory," Smart Structures and Systems, an International Journal, vol. 12, no. 3-4, pp. 345-361, 2013. 


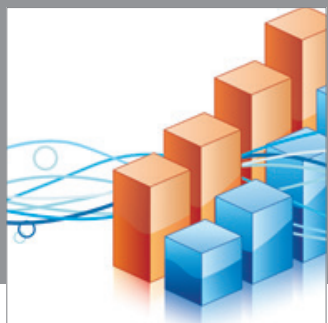

Advances in

Operations Research

mansans

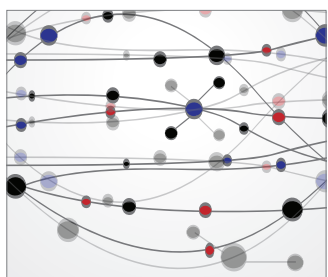

The Scientific World Journal
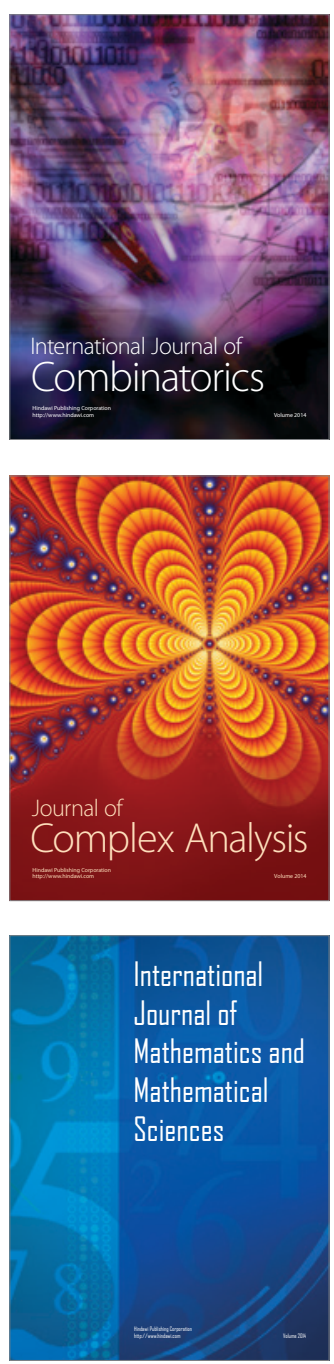
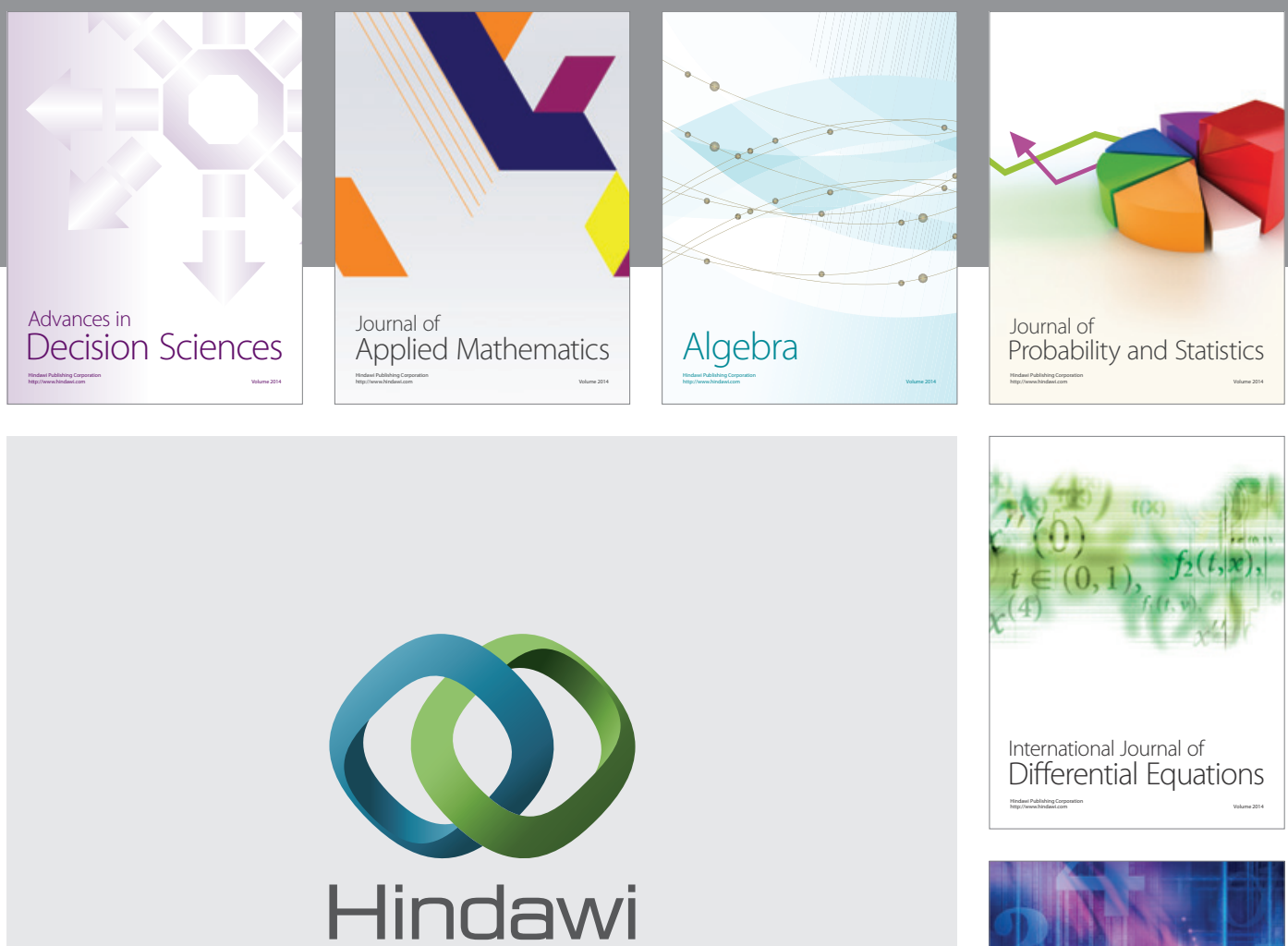

Submit your manuscripts at http://www.hindawi.com
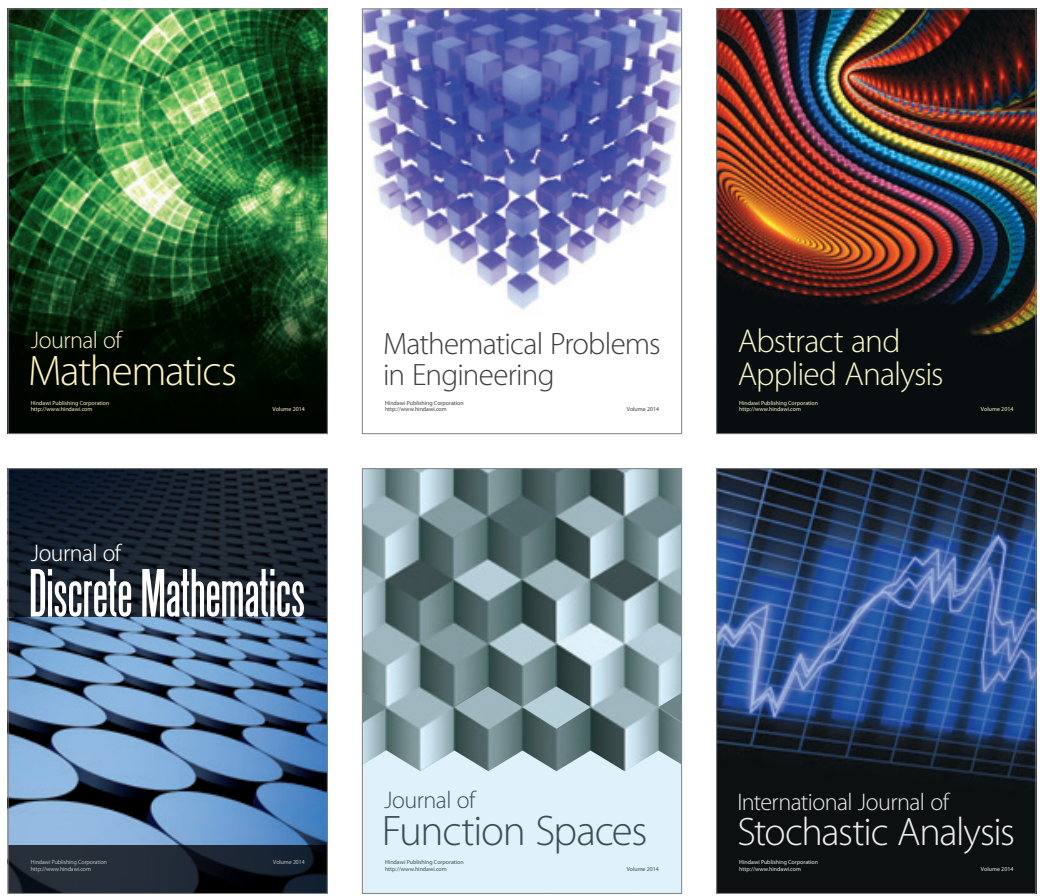

Journal of

Function Spaces

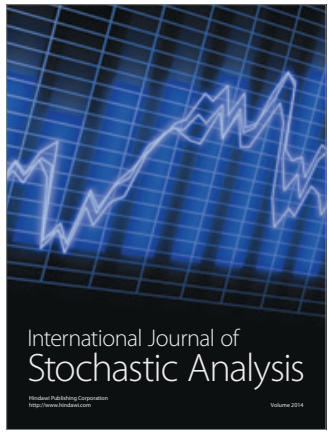

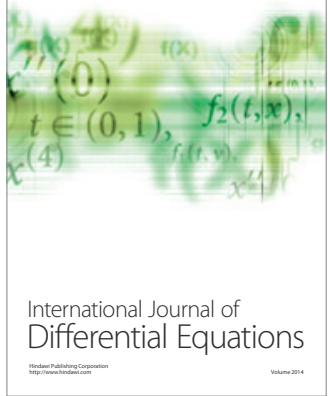
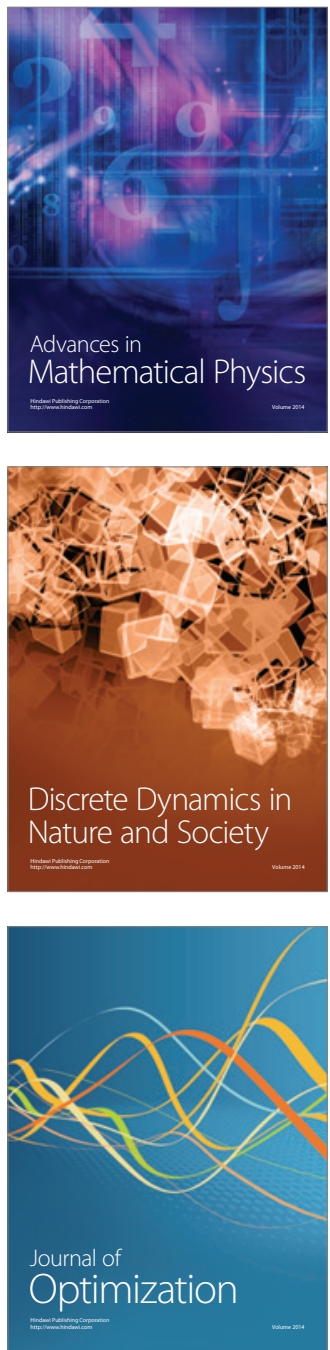